\title{
The Contrasting Roles of Planck's Constant in Classical and Quantum Theories
}

\author{
Timothy H. Boyer \\ Department of Physics, City College of the City \\ University of New York, New York, New York 10031
}

\begin{abstract}
We trace the historical appearance of Planck's constant in physics, and we note that initially the constant did not appear in connection with quanta. Furthermore, we emphasize that Planck's constant can appear in both classical and quantum theories. In both theories, Planck's constant sets the scale of atomic phenomena. However, the roles played in the foundations of the theories are sharply different. In quantum theory, Planck's constant is crucial to the structure of the theory. On the other hand, in classical electrodynamics, Planck's constant is optional, since it appears only as the scale factor for the (homogeneous) source-free contribution to the general solution of Maxwell's equations. Since classical electrodynamics can be solved while taking the homogenous source-free contribution in the solution as zero or non-zero, there are naturally two different theories of classical electrodynamics, one in which Planck's constant is taken as zero and one where it is taken as non-zero. The textbooks of classical electromagnetism present only the version in which Planck's constant is taken to vanish.
\end{abstract}




\section{INTRODUCTION}

"Planck's constant $h$ is a "quantum constant"' is what I am told by my students. "Planck's constant is not allowed in a classical theory" is the view of many physicists. I believe that these views misunderstand the role of physical constants and, in particular, the role of Planck's constant $h$ within classical and quantum theories. It is noteworthy that despite the current orthodox view which restricts the constant $h$ to quantum theory, Planck searched for many years for a way to fit his constant $h$ into classical electrodynamics, and felt he could find no place. Late in life, Planck[1] acknowledged that his colleagues felt that his long futile search "bordered on a tragedy." However, Planck's constant $h$ indeed has a natural place in classical electrodynamics. Yet even today, most physicists are unaware of this natural place, and some even wish to suppress information regarding this role. In this

article, we review the historical[2] appearance of Planck's constant, and then emphasize its contrasting roles in classical and quantum theories.

\section{PHYSICAL CONSTANTS AS SCALES FOR PHYSICAL PHENOMENA}

Physical constants appear in connection with measurements of the scale of natural phenomena. Thus, for example, Cavendish's constant $G$ appeared first in connection with gravitational forces using the Newtonian theory of gravity. At the end of the 18th century, Cavendish's experiment using a torsion balance in combination with Newton's theory provided the information needed for an accurate evaluation of the constant. However, because physical constants refer to natural phenomena and are not the exclusive domain of any one theory, Cavendish's constant $G$ also reappears in Einstein's 20th-century general-relativistic description of gravitational phenomena.

In a similar fashion, Planck's constant $h$ sets the scale of electromagnetic phenomena at the atomic level. It was first evaluated in 1899 in connection with a fit of experimental data to Wien's theoretical suggestion for the spectrum of blackbody radiation. Subsequently, the constant has reappeared both in quantum theory and in classical electrodynamics. In its role as a scale, the constant $h$ can appear in any theory which attempts to explain aspects of atomic physics. 


\section{PHYSICAL CONSTANTS OF THE 19TH CENTURY}

The 19th century saw developments in theories involving a number of physical constants which are still in use today. The kinetic theory of gases involves the gas constant $R$ and Avogadro's number $N_{A}$. The unification of electricity and magnetism in Maxwell's equations involves an implicit or explicit (depending on the units) appearance of the speed of light in vacuum $c$. Measurements of blackbody radiation led to the introduction of new physical constants. Thus the appearance of the Stefan-Boltzmann law $\mathcal{U}_{T}=a_{S} T^{4} V$ for the thermal energy $\mathcal{U}_{T}$ of radiation in a cavity of volume $V$ at temperature $T$ introduced a new constant $a_{S}$, Stefan's constant, in 1879. Today this constant is reexpressed in terms of later constants as $a_{S}=\pi^{2} k_{B}^{4} /\left(15 \hbar^{3} c^{3}\right)$. In the $1890 \mathrm{~s}$, careful experimental work on the spectrum of blackbody radiation led to Wien's theoretical suggestion for the blackbody radiation spectrum with its two constants, (labeled here as $\alpha$ and $\beta$ ),

$$
\rho_{W}(\nu, T)=\alpha \nu^{3} \exp [-\beta \nu / T]
$$

Also at the end of the century, the measurement of the ratio of charge to mass for cathode rays led to new constants involving the charge and mass of the electron.

\section{APPEARANCE OF PLANCK'S CONSTANT}

Planck's great interest in thermodynamics led him to consider the equilibrium of electric dipole oscillators when located in random classical radiation. Planck found that the random radiation spectrum could be connected to the average energy $U(\nu, T)$ of an electric dipole oscillator of natural frequency $\nu$ as $\rho(\nu, T)=\left(8 \pi \nu^{2} / c^{3}\right) U(\nu, T)$. Introducing Wien's suggestion $\rho_{W}$ in (11) for the spectral form, Planck found for the average energy of an oscillator

$$
U_{W}(\nu, T)=h \nu \exp [-\beta \nu / T]
$$

Here is where Planck's constant $h$ first appeared in physics. At the meeting of the Prussian Academy of Sciences on May 18, 1899, Planck reported[3] the value $\beta$ as $\beta=0.4818 \times$ $10^{-10} \mathrm{sec} \cdot \mathrm{K}^{o}$ and the value of the constant $h$ as $h=6.88510^{-27}$ erg-sec. Thus initially, Planck's constant appeared as a numerical fit to the experimental blackbody data when using theoretical ideas associated with Wien's proposed form for the radiation spectrum. Planck's constant $h$ had nothing to do with quanta in its first appearance in physics. 
In the middle of the year 1900, experimentalists Rubens and Kurlbaum found that their measurements of the blackbody radiation spectrum departed from the form suggested by Wien in Eq. (11). It was found that at low frequencies (long wavelengths) the spectrum $\rho(\nu, T)$ seem to be proportional to $\nu^{2} T$. Planck learned of the new experimental results, and, using ideas of energy and entropy for a dipole oscillator, introduced a simple interpolation between the newly-suggested low-frequency form and the well-established Wien high-frequency form. His interpolation gave him the Planck radiation form corresponding to a dipole oscillator energy

$$
U_{P}(\nu, T)=\frac{h \nu}{\exp [\beta \nu / T]-1} .
$$

Planck reported[4] this suggested blackbody radiation spectrum to the German Physical Society on October 19, 1900. The Planck spectrum (3) involved exactly the same constants as appeared in his work when starting from Wien's spectrum. The experimentalists confirmed that Planck's new suggested spectrum was an excellent fit to the data. Once again Planck's constant $h$ appeared as a parameter in a fit to experimental data when working with an assumed theoretical form. There was still no suggestion of quanta in connection with Planck's constant $h$.

Planck still needed a theoretical justification for his new spectral form. Although Planck had hoped initially that his dipole oscillators would act as black particles and would bring random radiation into the equilibrium blackbody spectrum, he had come to realize that his small linear oscillators would not change the frequency spectrum of the random radiation; the incident and the scattered radiation were at the same frequency. In late 1900 in an "act of desperation," Planck turned to Boltzmann's statistical work which he had earlier "vehemently rejected." It was in connection with the use of statistical ideas that Planck found that the constant $\beta$ in the radiation spectra could be rewritten as $\beta=h / k_{B}$ where $k_{B}=R / N_{A}$, where $R$ and $N_{A}$ were the constants which had already appeared in the kinetic theory of gases. Indeed, it was Planck who introduced Boltzmann's constant $k_{B}$ into physics. Boltzmann had always stated that entropy $S$ was proportional to the logarithm of probability $W$ without ever giving the constant of proportionality. Planck introduced the equality $S=k_{B} \ln W$. Also, in his calculations of the probability, Planck departed from Boltzmann's procedures in retaining the energy connection $\mathcal{E}=h \nu$ without taking the expected limit $\mathcal{E} \rightarrow 0$. Only by avoiding the limit, could Planck recover his radiation 
spectrum. It was here in retaining $\mathcal{E}=h \nu$, rather than taking Boltzmann's limit, that the association of Planck's constant $h$ with quanta first appeared.

\section{PLANCK'S CONSTANT IN CURRENT QUANTUM THEORY}

Although Planck's constant did not originally appear in connection with quantum theory, the constant became a useful scale factor when dealing with the photoelectric effect, specific heats of solids, and the Bohr atom. The theoretical context for all these phenomena was

quantum theory. Quantum theory developed steadily during the first third of the 20th century. Today quantum theory is regarded as the theory which gives valid results for phenomena at the atomic level.

Our current textbooks emphsize that quantum theory incorporates Planck's constant $h$ into the essential aspects of the theory involving non-commuting operators. Thus position and momentum are associated with operators satisfying the commutation relation $\left[\widehat{x}, \widehat{p}_{x}\right]=$ $i h /(2 \pi)=i \hbar$. The non-commutativity is associate with a non-zero value of Planck's constant $h$ and disappears when $h$ is taken to zero. Associated with the non-commuting operators is the zero-point energy $U=(1 / 2) h \nu_{0}$ of a harmonic oscillator of natural frequency $\nu_{0}$. The oscillator zero-point energy vanishes along with a vanishing value for $h$.

\section{PLANCK'S CONSTANT IN CLASSICAL ELECTRODYNAMICS}

The natural place for Planck's constant within classical electrodynamics is as the scale factor of the source-free contribution to the general solution of Maxwell's equations. Maxwell's equations for the electromagnetic fields $\mathbf{E}(\mathbf{r}, t)$ and $\mathbf{B}(\mathbf{r}, t)$ can be rewritten in terms of the potentials $\Phi(\mathbf{r}, t)$ and $\mathbf{A}(\mathbf{r}, t)$ where $\mathbf{E}=-\nabla \Phi-(1 / c) \partial \mathbf{A} / \partial t$ and $\mathbf{B}=\nabla \times \mathbf{A}$. In the Lorenz gauge, Maxwell's equations for the potentials become wave equations with sources in the charge density $\rho(\mathbf{r}, t)$ and current density $\mathbf{J}(\mathbf{r}, t)$,

$$
\begin{aligned}
& \left(\nabla^{2}-\frac{1}{c^{2}} \frac{\partial^{2}}{\partial t^{2}}\right) \Phi(\mathbf{r}, t)=-4 \pi \rho(\mathbf{r}, t) \\
& \left(\nabla^{2}-\frac{1}{c^{2}} \frac{\partial^{2}}{\partial t^{2}}\right) \mathbf{A}(\mathbf{r}, t)=-4 \pi \frac{\mathbf{J}(\mathbf{r}, t)}{c} .
\end{aligned}
$$


The general solutions of these differential equations in all spacetime with outgoing wave boundary conditions are

$$
\begin{aligned}
& \Phi(\mathbf{r}, t)=\Phi^{i n}(\mathbf{r}, t)+\int d^{3} r^{\prime} \int d t^{\prime} \frac{\delta\left(t-t^{\prime}-\left|\mathbf{r}-\mathbf{r}^{\prime}\right| / c\right)}{\left|\mathbf{r}-\mathbf{r}^{\prime}\right|} \rho\left(\mathbf{r}^{\prime}, t^{\prime}\right), \\
& \mathbf{A}(\mathbf{r}, t)=\mathbf{A}^{i n}(\mathbf{r}, t)+\int d^{3} r^{\prime} \int d t^{\prime} \frac{\delta\left(t-t^{\prime}-\left|\mathbf{r}-\mathbf{r}^{\prime}\right| / c\right)}{\left|\mathbf{r}-\mathbf{r}^{\prime}\right|} \frac{\mathbf{J}\left(\mathbf{r}^{\prime}, t^{\prime}\right)}{c},
\end{aligned}
$$

where $\Phi^{i n}(\mathbf{r}, t)$ and $\mathbf{A}^{i n}(\mathbf{r}, t)$ are the (homogeneous) source-free contributions to the general solutions of Eqs. (41) and (5). It is universally accepted that these are the accurate solutions. However, all the textbooks[5] of electromagnetism omit the source-free contributions $\Phi^{i n}(\mathbf{r}, t)$ and $\mathbf{A}^{i n}(\mathbf{r}, t)$, and present only the contributions due to the charge and currents sources $\rho(\mathbf{r}, t)$ and $\mathbf{J}(\mathbf{r}, t)$.

Within a laboratory situation, the experimenter's sources correspond to $\rho(\mathbf{r}, t)$ and $\mathbf{J}(\mathbf{r}, t)$ while the source-free terms $\Phi^{i n}(\mathbf{r}, t)$ and $\mathbf{A}^{i n}(\mathbf{r}, t)$ correspond to contributions the experimenter does not control which are present when the experimenter enters his laboratory. The terms $\Phi^{i n}(\mathbf{r}, t)$ and $\mathbf{A}^{i n}(\mathbf{r}, t)$ might correspond to radio waves coming from a nearby broadcasting station, or might correspond to thermal radiation from the walls of the laboratory. In a shielded laboratory held at zero temperature, there is still random classical radiation present which can be measured using the Casimir force[6] between two parallel conducting plates. Intrepreted within classical electromagnetic theory, this Casimir force responds to all the classical radiation surrounding the conducting parallel plates, and it is found experimentally[7] that this force does not go to zero as the temperature goes to zero. The Casimir force, interpreted within classical electromagnetic theory, indicates that there is classical electromagnetic zero-point radiation present throughout spacetime. The zero-point spectrum is Lorentz invariant with a scale set by Planck's constant $h$. Thus in a shielded laboratory at zero-temperature, the vector potential $\mathbf{A}(\mathbf{r}, t)$ should be written as

$$
\begin{aligned}
\mathbf{A}(\mathbf{r}, t) & =\sum_{\lambda=1}^{2} \int d^{3} k \widehat{\epsilon}(\mathbf{k}, \lambda)\left(\frac{h}{4 \pi^{3} \omega}\right)^{1 / 2} \sin [\mathbf{k} \cdot \mathbf{r}-\omega t+\theta(\mathbf{k}, \lambda)] \\
& +\int d^{3} r^{\prime} \int d t^{\prime} \frac{\delta\left(t-t^{\prime}-\left|\mathbf{r}-\mathbf{r}^{\prime}\right| / c\right)}{\left|\mathbf{r}-\mathbf{r}^{\prime}\right|} \frac{\mathbf{J}\left(\mathbf{r}^{\prime}, t^{\prime}\right)}{c}
\end{aligned}
$$

including a source-free contribution which is a Lorentz-invariant spectrum of plane waves with random phases $\theta(\mathbf{k}, \lambda)$ and with Planck's constant $h$ setting the scale. [8]

As indicated here, there is a natural place for Planck's constant $h$ within classical electromagnetic theory. Once this zero-point radiation is present in the theory, it causes zero-point 
energy for dipole oscillators, and it can be used to explain Casimir forces, van der Waals forces, oscillator specific heats, diamagnetism, the blackbody radiation spectrum, and the absence of "atomic collapse." [9]

\section{CONTRASTING ROLES FOR PLANCK'S CONSTANT}

Planck's constant $h$ sets the scale for atomic phenomena. However, the constant plays contrasting roles in classical and quantum theories. Within quantum theory, Planck's constant $h$ is embedded in the essential aspects of the theory. If one sets Planck's constant to zero, then the quantum character of the theory disappears; the non-commuting operators become simply commuting c-numbers, and the zero-point energy of a harmonic oscillator drops to zero.

On the other hand, within classical electrodynamics, Planck's constant does not appear in Maxwell's fundamental differential equations. Rather, Planck's constant appears only in the (homogeneous) source-free contribution to the general solution of Maxwell's equations. Thus classical electrodynamics can exist in two natural forms. In one form Planck's constant is taken as non-zero, and one can explain a number of natural phenomena at the atomic level. In the other form, Planck's constant is taken to vanish. It is only this second form which appears in the textbooks of electromagnetism and modern physics. However, even this form is quite sufficient to account for our macroscopic electromagnetic technology.

\section{COMMENTS ON PLANCK'S CONSTANT IN CLASSICAL THEORY}

Planck was a "reluctant revolutionary" who tried to conserve as much of 19th century physics as possible. Late in life Planck wrote[1] in his scientific autobiography, "My futile attempts to fit the elementary quantum of action somehow into the classical theory continued for a number of years, and they cost me a great deal of effort. Many of my colleagues saw in this something bordering on a tragedy." Within his lifetime, there seems to have been no recognition of a natural place for Planck's constant $h$ within classical electrodynamics. It was only in the 1960s, beginning with careful work by Marshall, [10] that it became clear that Planck's constant $h$ could be incorporated in an natural way within classical electrodynamics.

Despite the realization that Planck's constant is associated with atomic phenomena and 
that at least some of atomic phenomena can be described within either classical or quantum theory, there seems great reluctance on the part of some physicists to acknowledge the possibility of Planck's constant appearing within classical theory. One referee wrote the following justification [11] in rejecting the idea of Planck's constant appearing within classical electromagnetism: “But as a pedagogical matter, doesn’t it muddy the distinction between classical and quantum physics? The traditional dividing line may be in some aspects arbitrary, but at least it is clear $(\hbar \Longrightarrow$ quantum)." This referee clearly misunderstands the nature of physical constants and seems willing to sacrifice scientific accuracy to pedagogical simplicity.

I believe that many physicists would prefer truth to convenient pedagogy. Certainly the introduction of Planck's constant as the scale factor for source-free classical zero-point radiation expands the range of phenomena described by classical electromagnetic theory. [9] The recognition that Planck's constant has a natural place within classical theory may provide a broadening perspective beyond a confining quantum orthodoxy. In any case, one suspects that Planck would have been pleased to find that there is indeed a natural role for his constant $h$ within classical electromagnetic theory.

[1] M. Planck, Scientific Autobiography and Other Papers, translated by F. Gaynor (Philosophical Library, New York 1949), p. 35.

[2] The historical information is taken from the following sources: T. S. Kuhn, Black-Body Theory and the Quantum Discontinuity 1894-1912 (Oxford U. Press, New York 1978); A. Hermann (translated by C. W. Nash) The Genesis of Quantum Theory (1899-1913) (MIT Press, Cambridge, MA 1971); M. J. Klein, "Planck, Entropy, and Quanta 1901-1906," The Natural Philosopher I, pp.. 81-108 (Blaisdell Publishing Co. New York 1963); M. J. Klein, "Thermodynamics and Quanta in Planck's Work," in History of Physics edited by S. R. Weart and M. Philips (AIP, New York 1985), pp. 294-302.

[3] M. Planck, S.-B. Preuss. Akad. Wiss. (1899), p. 440.

[4] M. Planck, Verh. d. Deutsch. Phys. Ges. 2, 202 (1900).

[5] See for example, D. J. Griffiths, Introduction to Electrodynamics 4th ed. (Pearson, New York 2013), section 10.2.1 Eq. (10.26); or L. Eyges, The Classical Electrodynamic Field (Cambridge 
University Press 1972), p. 186, Eqs. (11.45) and (11.46); or J. D. Jackson, Classical Electrodynamics 3rd ed. (John Wiley \& Sons, New York, 1999), 3rd ed., p. 246, Eq. (6.48); or A. Zangwill, Modern Electrodynamics (Cambridge U. Press, 2013), p. 724-725, Eqs. (20.57) and (20.58); or A. Garg, Classical Electromagnetism in a Nutshell (Princeton U. Press, Princeton, NJ 08450, 2012), p. 204, Eqs.(54.16) and (54.17).

[6] H. B. G. Casimir, "On the attraction between two perfectly conducting plates," Proc. Ned. Akad. Wetenschap. 51, 793-795 (1948).

[7] M. J. Sparnaay, "Measurement of the attractive forces between flat plates," Physica (Amsterdam) 24, 751-764 (1958); S. K. Lamoreaux, "Demonstration of the Casimir force in the 0.6 to $6 \mu \mathrm{m}$ range," Phys. Rev. Lett. 78, 5-8 (1997): 81, 5475-5476 (1998); U. Mohideen, "Precision measurement of the Casimir force from 0.1 to $0.9 \mu \mathrm{m}$," ibid. 81, 4549-4552 (1998); H. B. Chan, V. A. Aksyuk, R. N. Kleinman, D. J. Bishop, and F. Capasso, "Quantum mechanical actuation of microelectromechanical systems by the Casimir force," Science 291, 1941-1944 (2001): G. Bressi, G. Caarugno, R. Onofrio, and G. Ruoso, "Measurement of the Casimir force between parallel metallic surfaces," Phys. Rev. Lett. 88, 041804(4) (2002).

[8] T. H. Boyer, "Random electrodynamics: The theory of classical electrodynamics with classical electromagnetic zero-point radiation," Phys. Rev. D 11, 790-808 (1975).

[9] T. H. Boyer, "Any classical description of nature requires classical electromagnetic zero-point radiation," Am. J. Phys. 79, 1163-1167 (2011). See also, D. C. Cole and Y. Zou, "Quantum Mechanical Ground State of Hydrogen Obtained from Classical Electrodynamics," Phys. Lett. A 317, 14-20 (2003). A review of the work on classical electromagnetic zero-point radiation up to 1996 is provided by L. de la Pena and A. M. Cetto, The Quantum Dice - An Introduction to Stochastic Electrodynamics (Kluwer Academic, Dordrecht 1996).

[10] T. W. Marshall, "Random electrodynamics," Proc. R. Soc. A276, 475-491 (1963). T. W. Marshall, "Statistical Electrodynamics," Proc. Camb. Phil. Soc. 61, 537-546 (1965).

[11] Author's personal correspondence.

(revised October 1, 2017) 